yeturned to its proper situation, but quickly slips forwards again when left to itself. As greater violence is required to produce these accidents than in simple dislocations; and, moreciver, as this violence must act directly upon the joint, you will have more contusion, more swelling, and considerably more pain than in the latter injuries. The result of these cases depends very much upon the situation of the fracture. When it occurs at the anatomical neck, or immediately below the tubercles, you will not be able to return the head of the bone to the glenoid cavity, as you will have no purchase whereby you can effect such reduction; but when the fracture obtains three inclies below the tubercles, as in the case related by M. Laumonier, or lower down, a cure may be effected, as we have already seen in that instance.

When, therefore, the fracture occurs at the anatomical neck, or immediately below the tubercles, extension is of no use, further than to bring the npper end of the shaft into the glenoid cavity.

Some difference of opinion has been expressed as to the treatment of these cases. Sir A. Cooper advises to keep the shaft in the axilla, " a pad placed in the latter, a clavicle bandage applied, and the elbow supported." Mr. Allan, of Edinburgh, observes, "When the fracture occurs below the tubercles, and above the insertion of the axillary muscles, the inferior portion of bone is always retracted upwards and inwards. Do not, there. fore, support the elbow."

I entirely agree with Mr. Allan as to the propriety of leaving the elbow unsupported. How so shrewd an observer as the late Sir A. Cooper, could have pointed out the propriety of extending the arm, to draw the shaft down to its proper place, and immediately afterwards lay down a line of conduct having a direct tendency to displace it, is eertainly somewhat surprising. It is true, that do what we may, the patient will never recover the proper use of his arm; the question, therefore, for our. consideration, is the degree of action which may be recovered; and our endeavours should be directed towards obtaining as great a latitude of motion as possible. I have already pointed out to you the extent to which the movements, especially the over-head movements, are impeded, we may say, entirely lestroyed, when the upper end of the shaft is drawn up against the coracoid process. It is, therefore, obvious that our treatment ought to be directed towards keeping the upper end of the shaft as much as possible in the glenoid cavity, and favouring the formation of adhesions, by which it may be permanently restrained in that situation. To support the elbow is consequently injudicious. I should recommend you to treat these accidents in the same way as you would a simple fracture of the anatomical neck of the humerus. To render this treatment the more effectual, I have invented a oplint, which is made by Mr. Simpson, of the Strand. You will perceive that it is an iron angular splint, with a joint where it corresponds to the elbow. It is intended to be placed on the inner side of the arm, and front of the forearm. That portion appropriated to the arm, consists of two pieces, which slide one upon the otber, and are tixed by a couple of thumb-screws, by which it may be adapted to any length of arm; whilst the upper or axillary end of the arm-piece ends in a pad, like the top of a crutch. This serves the treble purpose of counter-extension against the margins of the axilla ; preserving those margins from excoriation which the sharp edge of the splint might otherwise produce; and, lastly, it presents an axillary pad and splint in one, certainly an improvement upon the detached pad, which is always liable to shift and get out of its place. When the fracture has taken place lower down, more towards the middle of the shaft of the humerus, there can be no doubt that we should endeavour to reduce the dislocation at once, if we can, either by the plan adopted so successfully by M. Laumonier, or by one of the other methods which I shall have to describe to you hereafter.

With respect to reducing the dislocation, first, Bannister, in his " Chirurgery Digested," observes, "If a luxation happen with a fracture, the luxation, if it may be, is first, the fracture next, to be restored. If that cannot be, then compass the case of the fracture first, and after his callus is grown firm, undertake the luxation." This is very good advice, provided the circumstances of the case are favourable to reduction, and that the attempt may be made with safety, and probability of benefit to your patient; but we will discuss this point more fully by-and-bye.

PRACTICAL OBSERVATIONS ON SOME OF THE MORE IMPORTANT POINTS OF PHYSICAL DIAGNOSIS.

By C. M. Durraxt, M.D., Physician to the East Suffolk and Ipswich Hospital.

(Continued from page 18.)

\section{AUSCULTation OF THE LARYNX AND TRAChea.}

Before commenting on the phenomena referable to the voice, cough, \&c., as diagnostic of disease, it will be expedient to analyse very briefly the sounds proper to the commencing portions of the respiratory apparatus, viz., the larynx and trachea. These signs have been imperfectly investigated, and, indeed, by many have, intentionally, or otherwise, been overlooked. Nevertheless, as auxiliaries in diagnosis, their importance merits consideration.

In examining the region of the neck, the auscultator, while he maintains close proximity of the instrument to the surface, must press lightly, otherwise he will produce pain, and by disturbing the free current of air, render the result of the examination nugatory.

Both the normal and abnormal laryngo-tracheal sounds differ from those occurring in the chest, chieny by being more fully pronounced, dryer, more harsh, and of a somewhat abrupt whiffing character. Thus we have in disease, an exaggerated degree of the 
sonorous, sibilant, grating, whistling, and cavernous sounds, the pathological signification of which will be considered in detail, in connection with the physical diagnosis of the affections on which they severally obtain.

The physical causes to which the abnormal laryngeal sounds owe their origin, consist either in the transit of air through fluids contained in these passages, or in an altered condition of the cavities themselves, whether from a narrowed diameter, or from a thickened, ulcerated, and consequently uneven, state of the mucous membrane.

Although the phenomena elicited by auscultation of the larnyx and trachea, are wanting in the precision and definiteness which characterize those of the chest, still in many cases, a comparative investigation of the sounds produced in both localities becomes an important auxiliary in diagnosis. The differential value of these sounds in spasmodic affections of the air passages, foreign bodies in the larynx and trachea, tuberculous ulcerations, \&c., together with the character and degree of permanency which they may assume, renders the neglect of laryngeal auscultation, at least in all doubtful cases, highly culpable.

\section{AUSCULTATION OF THE VOICE.}

A knowledge of the various changes to which the resonance of the voice is subject in disease, becomes an important collateral aid in stethoscopic investigation. It will, however, be necessary that the student, in the first place, make himself acquainted with the natural characters of the vocal resonance, by the repeated auscultation of the chest of an individual in perfect health. With a full conviction of the importance of this latter suggestion, a few remarks indicative of some peculiarities in the natural vocal counds will be sufficient for our purpose.

In auscultating the voice, the sitting posture is to be preferred. Its tone and intensity should as nearly as possible be equally maintained while examining the two sides of the chest; to facilitate which, it is desirable to direct the patient to count a certain number of figures with a well-sustained and moderately loud utterance.

The circumstances modifying the resonance in a healthy state, are :-lst, the proximity to the trachea and the larger bronchi; 2 ndly, the natural tone of the individual; 3rdly, the thickness of the thoracic parietes. Thus, the nearer the instrument is applied to the seat of the larger air-tubes, the louder will be the resonance; the more grave the natural tone of the patient, the more tense will be the transmitted sound; and lastly, the more perfect the development of the chest, and the more free from fat, the louder will be the vocal reverberation. In all cases, however, a careful comparative examinution of the two sides of the chest sloould invariably be instituted.

The intensity of the rocal resonance, under the influence of disease, is not only liable to diminution and augmentation, but also to a complete modification in character. A diminished or suppressed condition of the vocal resonance is not of frequent occurrence, and only obtains in those rare cases, in which the conulucting power of the lung becomes decreased or lost by the intervention of air, as, soppetimes happens in extensive pneumo-thorax. Exaggerated reannance and bronchophony are, however, of frequent occurrence, and, with due care in reference to the analysis of individual diseases, become valuable adjuvants to successful diagnosis. As it is not, however, easy to determine, where natural vocal resonance terminates, and the exaggerated begins, it will be desirable to consider them as modifications of the same sound, differing only in degree.

Bronchophony is a clear, distinct, and unusually loud resonance of the voice within the chest, varying, as just remarked, in the dégree of its tone. When weak and feebly pronounced, it becomes necessary to examine carefully both sides of the chest, in order to substantiate a point of comparison on the healthy side. It is usually a permanent sound, so long as the lesion on which it depends exists, and is commonly associated with bronchial respiration. The physical cause of bronchophony consists either in an enlarged calibre of the bronchial tubes, or in an thnatural density of the pulmonary or adventitious tissues within the chest, becoming thereby better conductors of sound. The seat of bronchophony is variable; it may obtain in all parts of the chest.

Egophony is a sound, which, when once clearly heard is not liable to be mistaken; when not fully developed, its characters are more or less amalgamated with bronchophony. It is a clear, tremulous, and bleating sound, presenting many varieties of intonation, not easily described. The best definition of ægophony, is that of its resembling the peculiar and well-known voice imparted by the operator to the puppet "Punch." It is persistent, although liable to removal by change of posture. The physical cause of rgophony is the compression of the lung by a thin stratum of fluid, through which the vocal ribrations are communicated. It is usually detected in the lower part of the scapular region, and extending sometimes to the side. My own experience does not lead me to place implicit reliance on the diagnostic value of 'this sign, unless indeed it be unequirocally derelopea, and accompanied by other well-marked phenomena.

Pectoriloquy, in its perfect form, imparts the sensation of the voice issuing from a cavern in the lyng, and passing directly through the tube of the stethoscope with painful distinctness to the ear. This sound, from not having been perfectly understood, has, I am persuaded, very frequently been confounded with bronchophony, and hence have arisen, from time to time, errors in diagnosis; indeed so many circumstances are necessary, in order to ensure the production of perfect pectoriloquy, that, as a diagnostic sign for caverns in general, I prefer the substitution of "cavernous voice," as adopted by MM. Barth and Roger. In order that perfect pectoriloquy obtain, it is necessary that the cavity be of moderate size, superficial, communicating freely with the bronchi, empty, having smooth and dense walls, and free from interstitial bands. The existence of a cavity of this description favouring the vibration and transmission of the rocal resonance, constitutes the physical cause of true pectoriloquy. Although its favourite seat is the summit of one, or böth lungs, it may, when influenced by other lesions besides phthisis, occur indifferently in any part of the chest.

A modification of the two preceding sounds, in which the rocal phenomena assume a metallic character, constitutes the resonance termed amphoric. It 
has been aptly compared to the sound produced by speaking into an empty or partially-filled pitcher. In this, the voice is not articulate, as in pectoriloquy, neither does it give the impression as if passing through the tube of the stethoscope. The physical cause of amphoric vocal resonance is the existence of a large cavity in the lung, communicating with the bronchi, and filled, or nearly so, with air.

AUSCULTation of THE cough.

In auscultating the cough, it is desirable that precisely similar relations subsist between the observer and patient, in reference to posture, \&c., as when examining the voice and respiration. It is also equally necessary that the student familiarize his ear with the natural resonance of the cough, by repeated exploration of the chest of a bealthy person, carefully ensuring, that the patient, when directed, cough deeply, and not as is often the case with nervous individuals, confine the effort to the mouth and lips. Among the most valuable data furnished by the cough, is the ascertaining whether certain phenomena do, or do not, possess a persistent character. If, as sometimes happens, in consequence of a plug of viscid mucus, in a moderately sized bronchus, the vesicular murmur below this obstruction be entirely absent, rendering the diagnosis doubtful, it is evident that its removal by coughing will at once, by permitting free transit to the air, assist greatly in substantiating the true nature of the case. Other examples might readily be adduced, but these for the present may be profitably deferred.

Presuming, then, that the auscultator have acquired a perfect knowledge of the natural tussive resonance, we may proceed to the consideration of its pathological characters, which, like those of the voice, have been distinguished according to the sensations they impart, into bronchial, cavernous, and amphoric. These signs always accompany the corresponding phenomena of the voice and respiration, and to similar lesions are indebted for their physical cause and seat.

Bronchial cough consists of a resonance, abrupt, vibrating, and harsh, and appearing to originate in a portion of lung more or less immediately under the instrument.

Cavernous cough is loud, bollow, and puinfully ringing. It is transmitted with considerable force through the stethoscope, and reverberates, as it were, against the ear. Its characters, as expressive of its origin, are peculiarly distinctive.

Amphoric cough differs from the cavernous, in not being forcibly propelled through the tube of the instrument, but, like its counterpart, the vocal resonance, imparts the sensation of being generated in a large cavity, communicating with the bronchi. It is metallic, and resembles the sound produced by coughing into an empty pitcher.

In addition to the preceding signs, other sounds are revealed by auscultation, with the chardicters, and pathological import of which, it is necessary to be acquainted. These are, metallic tinkling, the sounds of friction, and the fluctuation of percussion.

Metallic tinkling, when once heard, is not liable to be confounded with any other auscultating phenomenon. It is a sound common to the respiration, roice,and cough,although it is both more constantly and more fully pronounced under the influence of the two latter acts, than the former. Its cluaracters are sharp and ringing, and well expressed by its definition; it closely resembles the sound elicited by striking a vessel of glass or metal with a pin. When metallic tinkling is audible during respiration, it almost invariably accompanies the movement of inspiration, and, as already stated, metallic tinkling very frequently co-exists with the amphoric phenomena above described. The physical cause of metallic tinkling has been the source of much discussion. Its origin is probubly two-fold; first, as it occurs in pneumo-hydro-thorax, with a pulmonary fistula opening below the level of the liquid: in this it is caused by the successive rising and bursting of bubbles of air on the surface of the liquid contained in the pleura, through which they are transmitted from the fistula. The second cause sometimes productive of this sound, is the descent of a drop of liquid from the upper part of the chest into the collection of fluid below, in consequence of sudden or violent motion, or on changing from the horizontal to the erect posture. More rarely the phenomena of tinkling may, under similar circumstances, be generated in the interior of a large and dense tuberculous excavation. Metallic tinkling, although not confined to, is most commonly audible in, the central or lateral regions of the chest, except when issuing from a tuberculous cavern, under which condition the apices of the lungs are of course most prone.

The sound of pleuritic friction, to which it is at present intended alone to refer, conveys the impression of a superficial, abrupt, and rubbing movement, arising from the gliding upon each other of two bodies, at an apparently short distance from the ear. It most commonly, although not invariably, coincides with the act of inspiration, requiring, in some cases, a forcible effort, or a cough, for its production. Pleuritic friction varies in its tone and degree of intensity, subject as we shall hereafter see, to the pathological lesion on which it depends. The extent of surface over which this sound is audible is in general limited; where very fully pronounced, it may cause a palpable fremitus; even to the extent of being felt by the patient himself. The physical cause of pleuritic friction is the attrition during respiration of the two surfaces of the pleuræ, rendered more or less rough by morbid deposit. In the healthy condition of this membrane the sound of collision between the pleuræ is inaudible. The ordinary seats of this sound are the central, lateral, or posterior regions of the chest; much more rarely the summit and base.

The simple allusion to the splashing sound elicited by succussion of the chest, for the production of which, it is necessary that both air and liquid be present, terminates the category of the most important auscultatory phenomena, which, for practical purposes, avail us so largely in the formation of a correct diagnosis in diseases of the respiratory organs; in studying which, however, the student cannot have too often or too forcibly impressed upon his mind, the necessity of what has already been insisted on, viz., examining slowly, and carefully; listening to gne sound at a time; the avoidance of a preconceived judgment; caution in expressing a too hasty opinion; the non-attention to which simple rules has too frequently afforded to the sceptic, apparent grounds, however untenable, for his perseverance in rejecting 
the valuable auxiliaries derivable from this mode of examination.

The abnormal phenomena revealed by percussion are comparatively limited, but in conjunction with the auscultatory signs just enumerated, afford indications at once precise and important. Independently of the variations in tone and intensity of the sounds elicited by tapping the surface of the chest, a considerable modification of their character frequently obtains, the peculiarities of which it will be necessary to mention. These have been chiefy distinguished into the tubular, tympanitic, and cracked-metal sounds.

The tubular character of the stroke sound, as its name implies, is caused either by the proximity of the larger bronchi to the surface, or by the intervention of a superimposed conducting medium, whether fluid or solid. It may be imitated by percussing the trachea.

The tympanitic sound which is sometimes elicited by percussion, arises from the existence of an abnormal quantity of air contained within the chest; at the same time the thoracic walls are commonly rendered more tense, by the filling up of the intercostal spaces.

The last phenomenon is the bruit de pot fêle or cracked metal sound, which when fully pronounced is extremely characteristic, and indicates a pulmonary cavity of considerable magnitude, lying near the surface of the lung.

In the foregoing résumé of physical signs, it will be observed that I have throughout endeavoured to maintain a close connection between them, and the pathological lesions from which they originate, since it is by this alone, that the auscultator can, with any degree of certainty, frame a diagnosis, that will in reference to treatment prove serviceable to his patient, or creditable to himself.

(To be continued.)

\section{ON DETACHING THE PLACENTA IN CASES OF PLACENTA PREVIA.}

By Thomas Radpord, M. D.,

Consulting Physician to the Mauchester and Salford Lying-in Hospital.

Since my observations on galvanism in uterine hæmorrhage were published in the Provincial Medical and Surgical Journal, I have had letters from many gentlemen, enquiring whether I confined the practice of detaching the placenta in cases of placenta provia to those of exhaustion alone. In order, then, to supersede the necessity of writing to each correspondent, I make the reply through the same channel. In my paper I stated that I had detached the placenta in a case which occurred in 1819 , but did not then state that it was unattended by exhaustion. From this and other cases then alluded to, I conclude, that on a complete separation of the placenta, the hæmorrhage is immediately and completely suppressed, provided the uterus is in a condition to so far contract, as to force down the head with the placenta upon the nterine openings. By this practice it may be said that the life of the child is sacrificed : but this will not always happen. We find from hospital and individual reports, that the child is usually dead when the case has been treated by the present recognized means.

In nearly all the cases which I have collected and referred to in my paper, of expulsion of the placenta by the natural efforts, we find that the mother recovered; and when this fortunate event did not happen, it depended upon the serious impression made upon the vital powers before the placenta was completely detached.

It may also be stated that uterine phlebitis takes place more frequently in cases of placenta previa, when the ordinary practice is adopted, than we observe in the same number of cases of accidental hæmorrhage. This result, in the opinion of the writer, arises from the contusion and slight lacerations which are consequent upon a forced delivery.

From the above statement, I consider I am justified in recommending a modified practice; but I shall not enter fully into the details of the plan, as this brief communication will not allow of it.

First. Then, as neither delivery, nor detaching the placenta, ought ever to be attempted until the cervix and os uteri will safely allow the introduction of the hand, rest, the application of cold, but, above all, the use of the plug must never be omitted in cases where they are respectively required.

Secondly. If there are unequivocal signs of the child's death, the placenta is to be completely detached, and the membranes are to be ruptured. The case is then to be left to the natural efforts, provided there be suffcient uterine energy; if otherwise, the ordinary means are to be used, and, in addition, galvanism.

Thirdly. When a narrow pelvis exists in connection with placenta provia, the practice is to detach the placenta and to remove it, then to perforate the head as soon as the condition of the parts allow, and to extract it by means of the crotchet.

Fourthly. When the os uteri is partially dilated, and dilatable so as to allow the easy introduction of the hand, when the membranes are ruptured, and strong uterine contraction exists, the practice is to detach the placenta completely.

Fifthly. In all cases of exhaustion, as already referred to in my paper, the practice is to draw off the liquor amnii, by perforating the placenta, ws then recommended, then to detach completely this organ, and apply galvanism.

Sixthly. - In all cases of partial presentation of the placenta, the artificial rupture of the membranes will generallybe found sufficient to arrest the hæmorrhage, but if that should prove ineffectual, then we must apply galvanism.

The practice of detaching and removing the placenta was adopted by some of the older writers; and as I have mentioned in my paper ' On galvanism applied to the treatment of uterine hæmorrhage,' I detached this organ in the year 1819, although it was not my custom to do so.

Early in October, I received a letter from my respected friend Professor Simpson, in which he stated he removed the placenta in a case of unavoidable hæmorrhage. He "had the placenta on a plate two hours before the baby was born." The mother recorered. Dr. Simpson has collected a great number of cases of expulsion of the placenta before the child, and has come to the conclusion, that the practice of its remoral in some cases of placents provia, is calculated to sare the patient's life.

I am glad to hare my views on this most important 